\title{
First- and last-passage Monte Carlo algorithms for charge density on a conducting surface
}

\author{
Chi-Ok Hwang ${ }^{1, a}$, James A. Given ${ }^{2}$, Youngwon $\mathrm{Kim}^{3}$, Sunggeun Lee ${ }^{4}$ and Sungbae Lee ${ }^{1}$ \\ ${ }^{1}$ Division of Liberal Arts and Sciences, GIST College, Gwangju Institute of Science and Technology (GIST), \\ Gwangju, South Korea \\ ${ }^{2}$ Naval Research Lab., MS5720, 4555 Overlook Avenue SW, Washington, DC 20375, USA \\ ${ }^{3}$ Department of Physics and Photon Science, Gwangju Institute of Science and Technology (GIST), Gwangju, South \\ Korea \\ ${ }^{4}$ School of Electrical Engineering and Computer Science, Gwangju Institute of Science and Technology (GIST), \\ Gwangju, South Korea
}

\begin{abstract}
First-passage and last-passage algorithms, which are two diffusion Monte Carlo methods, can obtain charge density on a conducting surface. In general, the first-passage algorithms have been used to obtain the capacitance of the arbitrary-shaped conductors. In contrast, the last-passage algorithm was introduced to calculate the charge density at a general point on a conducting surface by using the diffusing paths that initiate at that point. Here, the conductor was held at unit voltage without any charge outside of the conductor. The two algorithms are inherently related. The last-passage algorithm is the time reversal of the firstpassage algorithm. In this paper, it is shown that Kai Lai Chung's last-passage algorithm is equivalent to the first-passage algorithm. In addition, based on the time reversality of the lastpassage algorithms we extend the last-passage algorithm to calculate the charge density with a charge distribution and a dielectric interface also. Lastly, we mention the recent progress in which we can obtain the charge density on a conducting surface under the general non-constant Dirichlet boundary conditions.
\end{abstract}

Keywords: monte carlo; first-passage; last-passage; charge density.

\section{Introduction}

According to the probabilistic potential theory [1, 2], there is a one-to-one correspondence between an elliptic partial differential equation and a diffusion process. Using the correspondence, Monte Carlo diffusion algorithms have been developed to solve some elliptic partial differential equations [3-6]. Elliptic partial differential equations such as Laplace and Poisson equations are governing equations for charge density problems. First-passage and last-passage algorithms [3, 5, 6], which are two diffusion Monte Carlo methods, can obtain the charge density on a conducting surface. These two algorithms are inherently related. The last-passage algorithm is the time reversal of the first-passage algorithm.

In some applications such as charge singularity problems for parasitic capacitance in nano-scale interconnects, binding-site problems, problems involving singularities and so on, last-passage algorithms have an advantage over the first-passage algorithms. Last-passage algorithms can give the

a Corresponding author : chwang@gist.ac.kr 
charge density at a specific point on the surface whereas first-passage algorithms provide the charge distribution over the entire surface [7].

In previous researches [7, 8], a last-passage algorithm was introduced to calculate the charge density at a point on a conducting surface by using the diffusing paths that initiate at that point. At that time, the conductor was held at unit voltage without any charge outside of the conductor.

In this paper, it is shown that Kai Lai Chung's last-passage algorithm is equivalent to the firstpassage algorithm. In addition, based on the time reversability of the last-passage algorithms we extend the last-passage algorithm to calculate the charge density with a charge distribution and a dielectric interface also. Lastly, we mention the recent progress in which we can obtain the charge density on a conducting surface under the general non-constant Dirichlet boundary conditions.

\section{First-passage algorithms}

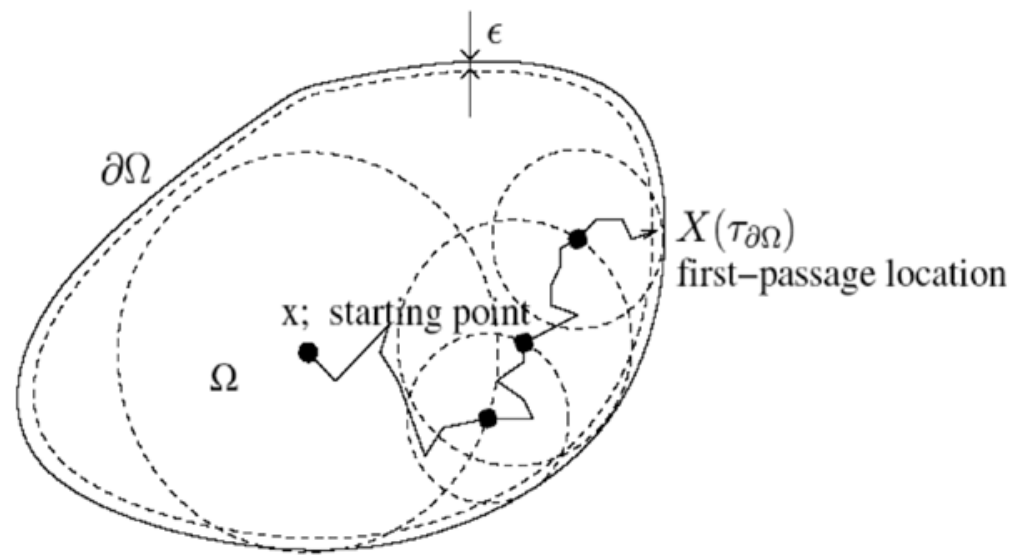

Figure 1. Schematic view that illustrates a first-passage jump using "walk on spheres” (WOS) algorithm. In WOS, there is an $\varepsilon$-layer. A Brownian walk is simulated using a series of discrete jumps using WOS spheres until the walk is terminated in the layer.

The probabilistic potential theory $[1,2]$ states that the solution to the Laplace boundary value problem,

$$
\nabla^{2} f(\mathbf{x})=0, \quad \mathrm{x} \in \Omega,
$$

with the boundary conditions $f(\mathbf{x})=\psi(\mathbf{x})$, when $\mathbf{x} \in \partial \Omega$, is

$$
f(\mathbf{x})=E\left[\psi\left(\mathbf{x}_{\tau}\right)\right] .
$$

Here, $\tau$ is the first-passage time. From the statistical viewpoint, the solution can be reinterpreted as an average of the boundary values,

$$
f(\mathbf{x})=\int_{\partial \Omega} p(\mathbf{x}, \mathbf{y}) \psi(\mathbf{y}) d \mathbf{y} .
$$

Here, $p(\mathbf{x}, \mathbf{y})$ is the first-passage probability distribution function when $\mathbf{x}$ is the starting location of the random walker. Another electrostatic solution representation using Green's function, $G(\mathbf{x}, \mathbf{y})$, is

$$
f(\mathbf{x})=\int_{\partial \Omega} \frac{\partial G(\mathbf{x}, \mathbf{y})}{\partial \mathbf{n}} \psi(\mathbf{y}) d \mathbf{y} .
$$

Here, the electrostatic boundary is considered as a perfect conductor and $\partial G(\mathbf{x}, \mathbf{y}) / \partial \mathbf{n}$ considered as 
the induced surface charge density in electrostatics.

From Eqs. 3 and 4, we can understand the isomorphism between the electrostatic induced surface charge density and the first-passage probability distribution; that is, this shows the basis for the Green's function first-passage (GFFP) algorithms [4]. We have been using many boundary geometries, such as sphere ("Walks on Spheres" [WOS] algorithm (see Fig. 1) and "Off-centered WOS" [5]), infinite plane ("Walks on Planes" [WOP] algorithm) (see Fig. 2), spherical surfaces when the diffusion random walker is outside the sphere (see Fig. 3) and spherical surfaces chopped by a flat boundary (GFFP algorithms). In the WOS algorithm, an $\varepsilon$-layer is introduced to terminate the random walk. The error associated with the $E$-layer was investigated in [9].

Usually, first-passage algorithms are used for the calculation of capacitances, which can be obtained by computing the probability (relative capacitance with respect to the launching sphere) of a random walk started on the launching sphere [10]. This means that the summation of all the induced charge density over the target object can give the capacitance of the object. The capacitance of the unit cube has been extensively calculated in this way [11-15].

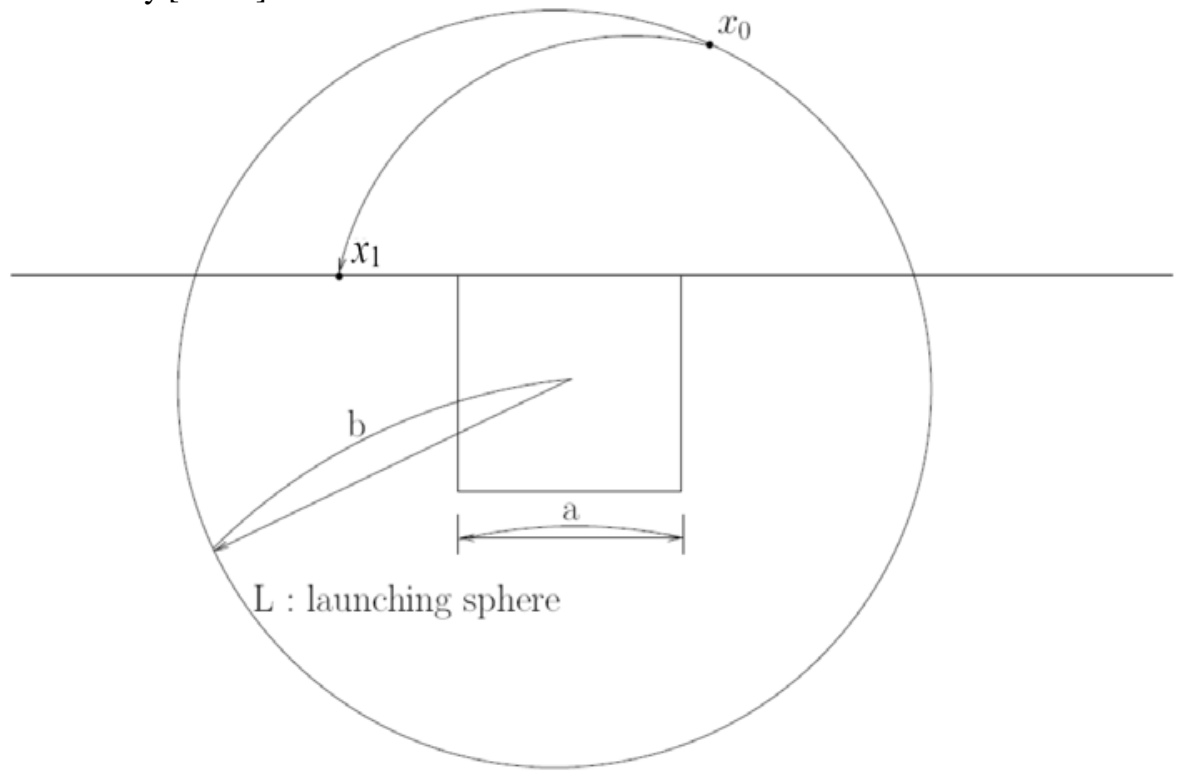

Figure 2. Schematic side view that illustrates a first-passage jump using the walk-on- planes (WOP) algorithm [6]. In the WOP, there is no $\varepsilon$-layer. A Brownian walk is initiated with uniform probability on the launching sphere, $L$ of radius $b$, and proceeds by either striking an infinite plane that includes one of the six surfaces of the unit cube $(\mathrm{a}=1)$ and is declared to be absorbed landing on the surface of the unit cube, or falls on the plane outside of the cube surface, in which case the walk continues [8].

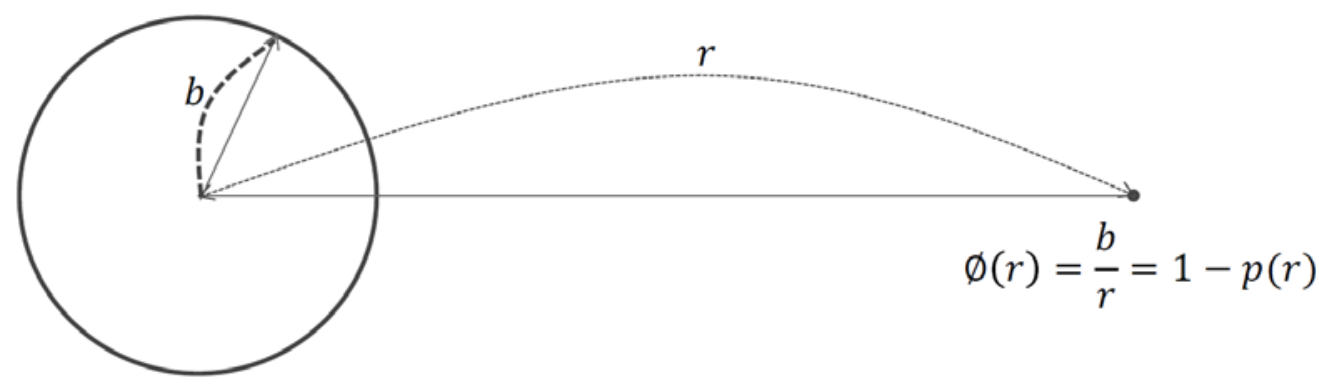

Figure 3. Schematic diagram for the relation of potential in electrostatics and the probability of the Brownian particle starting from distance $\boldsymbol{r}$ returning to the sphere. The probability $p(\boldsymbol{r})$ is the probability of going to infinity without returning to the sphere. 


\section{Equivalence of Kai Lai Chung's last-passage algorithm to the first- passage algorithm}

Here, we show that Kai Lai Chung's last-passage algorithm is equivalent to the first-passage algorithm. It is well known that the surface charge distribution on a conductor held at a nonzero potential with respect to infinity can be obtained by a first-passage algorithm [6, 8].

$$
\sigma(d y)=F(\infty ; d y)
$$

where $F(\infty ; d y)$ is the first-passage probability distribution of the Brownian motion starting from infinity. In practice, the simulation of the Brownian motion starting from infinity can be conducted using an enclosing sphere of the object, where the Brownian particle can be launched uniformly (see Fig. 2). In addition, we can estimate the capacitance of the object from the total charge $[3,6,8]$.

In contrast, according to Kai Lai Chung's book [16], the charge density is given by

$$
\sigma(d y)=2 \pi\|\mathbf{x}-\mathbf{y}\| L(x ; d y),
$$

here, $L(x, d y)$ is the last-passage density of the Brownian motion starting from $\mathbf{x}$ and going to infinity.

There is time-reversal symmetry of the Brownian motions of the first-passage algorithm starting from infinity to $\mathbf{x}$ and of Chung's last-passage algorithm starting from $\mathbf{x}$ to infinity (See Fig. 4.). The first-passage location of the first-passage algorithm becomes the last-passage location of Chung's lastpassage algorithm. By equating the previous two equations, the last-passage probability distribution of Chung's algorithm can be obtained from the first-passage probability distribution starting from infinity to $\mathbf{x}$. That is,

$$
L(x ; d y)=\frac{1}{2 \pi\|\mathbf{x}-\mathbf{y}\|} F(\infty ; d y) .
$$

There is a finite probability that some of the trajectories of the first-passage starting from infinity will go to the small sphere centered at point $\mathbf{x}$. We should note that in practice we should use a small sphere to overcome the conceptual problem that there is zero probability that the trajectory of the

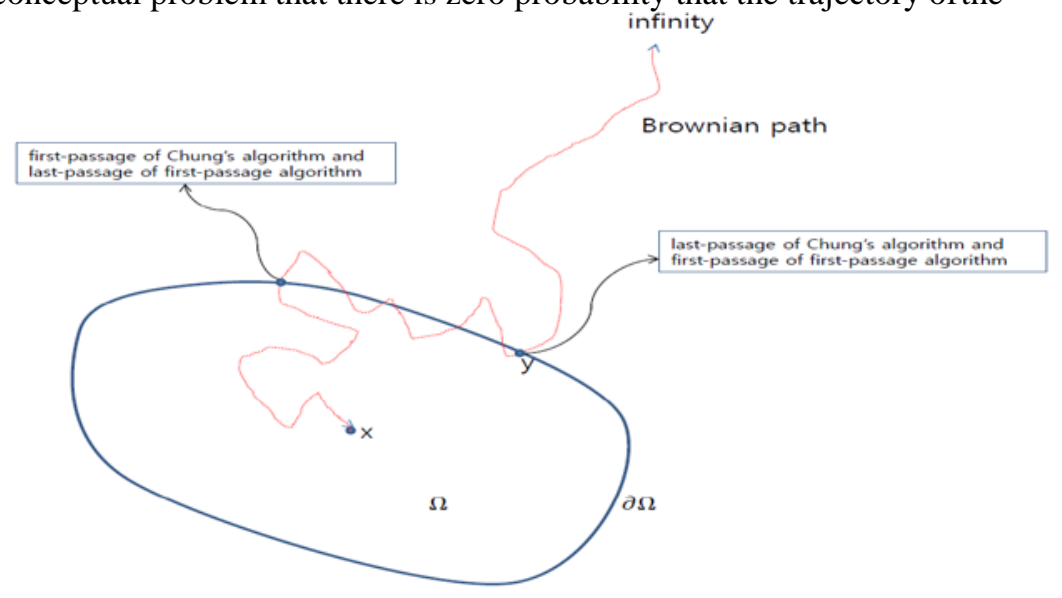

Figure 4. Schematic diagram for the equivalence of Chung's last-passage trajectory to the first-passage trajectory starting from infinity to point $\mathbf{x}$ : time reversal of the Brownian trajectory of the first-passage algorithm gives the trajectory of Chung's last-passage algorithm.

First-passage starting from infinity will go to point $\mathbf{x}$. The other trajectories of the first-passage starting from infinity to the first-passage point on the boundary go to infinity without going to the 
small sphere centered at point $\mathbf{x}$. In the first-passage algorithm after the first-passage on the boundary y from infinity, there is a finite probability, In the first-passage algorithm after the first-passage on the boundary $\mathbf{y}$ from infinity, there is a finite probability, $1 /(2 \pi|\mathbf{x}-\mathbf{y}|)$, that the Brownian particle will go to the small sphere centered at the point $\mathbf{x}[11,18]$ (see Fig. 4.). From this, we understand the Chung's last-passage algorithm.

Using the equivalence and the first-passage distribution, we can obtain the last-passage distribution. In addition, Chung's algorithm can be advantageous for the local charge distribution such as edge or corner charge distribution exponent calculations. However, we should note that from the practical point of simulations for the overall charge distribution on the conductor and the capacitance, the firstpassage algorithm is much more convenient than Chung's last-passage algorithm. Chung's last- passage algorithm was developed to Given-Hwang's last-passage algorithm [8, 17, 19] in the next section. In the Given-Hwang's last-passage algorithm, $\mathbf{x}$ and $\mathbf{y}$ in the Chung's last-passage algorithm coincide.

\section{Extended last-passage algorithms}

\subsection{Introduction}

In the previous research $[7,8]$, we treated a simple Laplace problem in which there is a conductor held at unit potential in a charge-free space (see Fig. 5(a)). Using the last-passage algorithm, we can obtain the charge density at a point on the conducting surface as follows:

$$
\sigma(\mathbf{x})=\frac{1}{4 \pi} \int_{\partial \Omega_{y}} d^{2} \mathbf{y} G(\mathbf{x}, \mathbf{y}) p(\mathbf{y}, \infty)
$$

For a flat conducting surface, the dipole Green's function is [6]

$$
G(\mathbf{x}, \mathbf{y})=\frac{3}{2 \pi} \frac{\cos \theta}{a^{3}},
$$

where $\theta$ is the angle between the vectors $\mathbf{x}$ and $\mathbf{y}$, and $a$ is the radius of the absorbing sphere. This dipole Green's function is rederived in Appendix A.

Here, we extend the last-passage algorithms to the electrostatic problems, including a charge and a dielectricinterface.

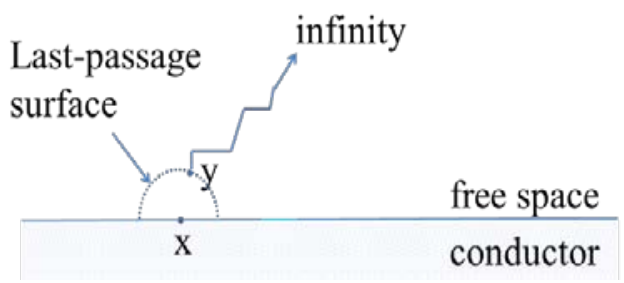

(a)

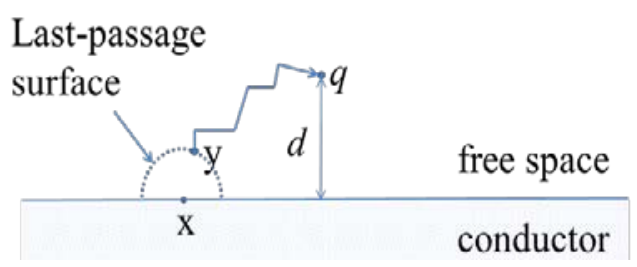

(b)

Figure 5. S Schematic diagram for the last-passage algorithm for the charge density on a conducting surface (a) held at a unit voltage (b) with a charge.

\subsection{Charge density with charge distribution}

First, we deal with the electrostatic point-charge problem. In this case, using the first-passage algorithms, we can obtain the charge density as follows. We can initiate random walkers from the point charge until the random walker reaches the conductor or goes to infinity. The last-passage algorithm is the time reversal of the first-passage algorithm [3,4], using which we can devise the last-passage 
algorithm. We initiate random walkers from the specific point where we want to know the charge density. The random walker walks until it reaches to the charge point (in practice, a small sphere centered at the point in the computer simulation), returns to the charge conductor or goes to infinity. There is a finite probability that the Brownian particle will go to the small sphere $[11,18]$.

$$
\sigma(\mathbf{x})=\frac{1}{4 \pi} \int_{\partial \Omega_{y}} d^{2} \mathbf{y} G(\mathbf{x}, \mathbf{y}) p\left(\mathbf{y}, \mathbf{y}^{\prime}\right) .
$$

We can understand this new last-passage algorithm with a charge such that in the last-passage algorithm for the charge density on a conducting surface held at unit voltage without any charge outside of the conductor, the charge can be thought to be away at the infinite point. In the new last passage algorithm with a charge, the charge is moved to a finite distance.

As an illustration, we consider the surface-charge density on a conducting plane of infinite extent induced by a charge at a distance $d$ from the surface (see Fig. 5(b)). The charge density is analytically known to be as follows: [20]

$$
\sigma=\frac{q d}{2 \pi} \frac{1}{\left(r^{2}+d^{2}\right)^{3 / 2}} .
$$

Here, $r$ is the radial distance from the intersection point where the distant line and the plane meet. In Fig. 5(b), using the last-passage algorithm we obtained the charge density on an infinite and flat conducting surface with charge outside the surface. We plotted charge density $v$.s. The radial distance from the charge (refer to Fig. 6) and could obtain radial charge density distribution the same as that in the analytic results.

\subsection{Charge density on a dielectric interface}

Next, we consider the charge density on a dielectric surface with a charge (see Fig. 7). In this case, the analytic solution for the charge density is as

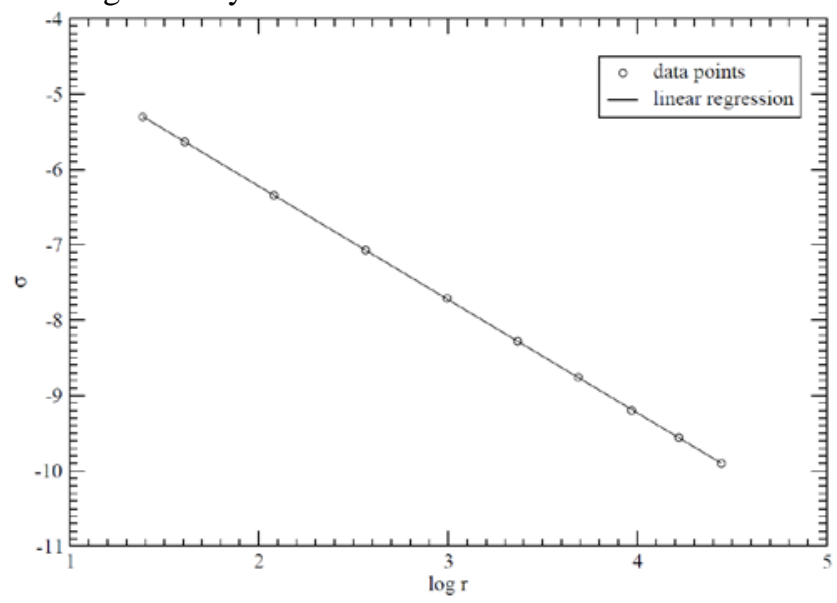

Figure 6. Charge density on the infinite and flat conducting surface vs. radial distance from the charge. The linear regression slope for the solid line is -1.5038 with determination coefficient -0.999986 . 


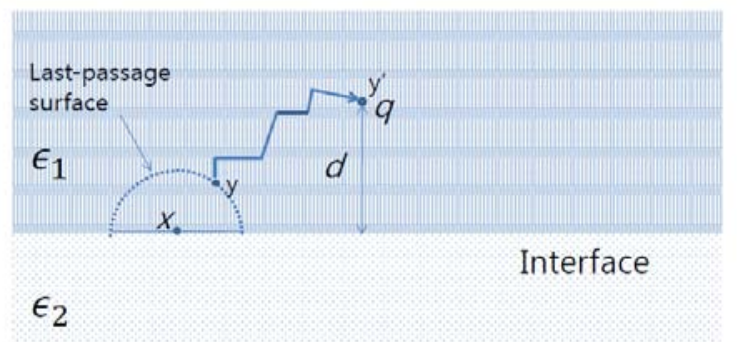

Figure 7. Schematic diagram for the last-passage algorithms for the charge density at $\mathbf{x}$ on the interface of two dielectric materials, $\varepsilon_{1}$ and $\varepsilon_{2}$ respectively, with charge $q$ at distance $d$ from the interface.

Follows [21]

$$
\sigma=\frac{q d \epsilon_{0}}{2 \pi \epsilon_{1}} \frac{\left(\epsilon_{1}-\epsilon_{2}\right)}{\left(\epsilon_{1}+\epsilon_{2}\right)} \frac{1}{\left(r^{2}+d^{2}\right)^{3 / 2}} .
$$

The only difference from the previous case is the proportionality constant involved in the dielectric coefficients. This shows that it is sufficient to understand the proportionality constant.

It is noted that the probability of $p_{1}$ landing on the dielectric side with $\varepsilon_{1}$ from the interface is proportional to thelocalconductivity of the corresponding side [22].

$$
p_{1}=\frac{\epsilon_{1}}{\left(\epsilon_{1}+\epsilon_{2}\right)}
$$

This explains the proportionality constant involved in the dielectric coefficients. Combined with the first free-space case, we can understand the analytic solution.

\subsection{Charge density under the general non-constant Dirichlet boundary conditions}

Recently the last-passage algorithms have been further developed to include the general non-constant Dirichlet boundary conditions [19]. The correction term in the last-passage algorithm of Eq. 4 due to the non-constant Dirichlet boundary conditions is as follows:

$$
-\frac{1}{2 \pi} \int_{S_{a} \backslash \Lambda_{\delta}} \frac{1}{a^{3}}(\phi(y)-\phi(x)) d s_{y}+O(\delta),
$$

with a circular patch $\Lambda \delta$ of radius $\delta$ centered at $x$. Here, the integration is over the ring-shaped region, $S_{a} \backslash \Lambda \delta$, with an accuracy of $O(\delta)$.

\section{Conclusions}

The first-passage and last-passage algorithms can obtain charge density on a conducting surface. While the first-passage algorithms have been used to obtain the capacitance of the arbitrary-shaped conductors in general, the last-passage algorithm was used to calculate the charge density at a general point on a conducting surface by using the diffusing paths that initiate at that point. Here, the conductor was held at unit voltage without any charge outside of the conductor.

The two algorithms are inherently related. The last-passage algorithm is the time reversal of the first-passage algorithm.In this paper, it is shown that Kai Lai Chung's last-passage algorithm is equivalent to the first-passage algorithm. In addition, based on the time reversability of the lastpassage algorithms we extended the last-passage algorithm to calculate the charge density with a 
charge distribution and a dielectric interface also. Lastly, we mention the recent progress in which we can obtain the charge density on a conducting surface under the general non-constant Dirichlet boundary conditions.

\section{Acknowledgements}

This research was supported by the Basic Science Research Program through the National Research Foundation of Korea (NRF), funded by the Ministry of Education, Science and Technology (Research fund number: 2013026313, 2012R1A1A2003025) and "Research support projects for distinguished professors” through a grant provided by GIST in 2016 (Grant No. GK03872). In addition, this research has been performed as a collaborative research effort of project No (Supercomputing infrastructure service and application) and supported by the KOREA INSTITUTE of SCIENCE and TECHNOLOGY INFORMATION (KISTI).

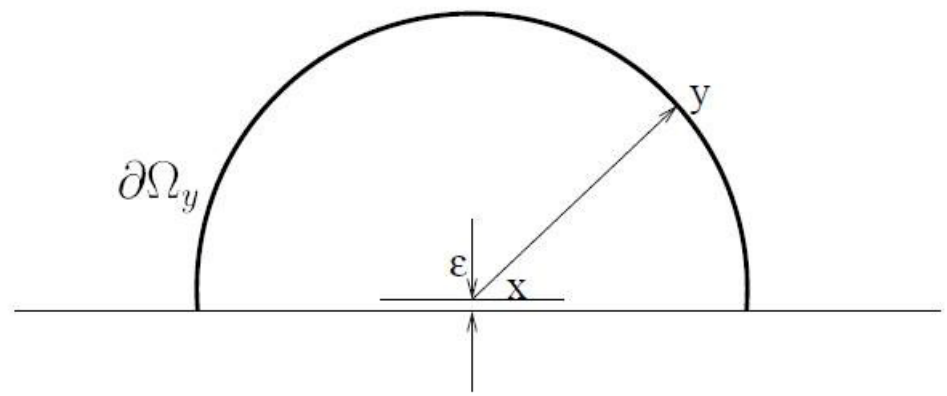

Figure 8. A conducting surface is shown edge on; $g(\mathbf{x}+\varepsilon, \mathbf{y})$ is the probability density associated with a Brownian particle initiating at the point $\mathbf{x}+\varepsilon$ and making first-passage on the surface $\partial \Omega y$ at the point $\mathbf{y}$ [8].

\section{Appendix}

In this appendix, we show the derivation of the Laplacian Green's function, $G(\mathbf{x}, \mathbf{y})$, for a point dipole centered on the conducting surface at point $\mathbf{x}$ and normal to the surface (see Fig. 9). The potential $V(\mathbf{y})$ $=V(r, \theta, \varphi)$ due to the dipole charge at $\mathbf{x}+\varepsilon$ and $\mathbf{x}-\varepsilon$ is given by

$$
V(r, \theta, \phi)=\frac{1}{4 \pi}\left(\frac{1}{\sqrt{r^{2}-2 \epsilon r \cos \theta+\epsilon^{2}}}-\frac{1}{\sqrt{r^{2}+2 \epsilon r \cos \theta+\epsilon^{2}}}\right) .
$$

By differentiating partially with respect to $r$, weobtain

$$
\frac{\partial V}{\partial r}=\frac{1}{4 \pi}\left(\frac{-r+\epsilon \cos \theta}{\left(r^{2}-2 \epsilon r \cos \theta+\epsilon^{2}\right)^{3 / 2}}+\frac{r+\epsilon \cos \theta}{\left(r^{2}+2 \epsilon r \cos \theta+\epsilon^{2}\right)^{3 / 2}}\right) .
$$

By differentiating partially with respect to $\varepsilon$ again and setting $\varepsilon=0$, we obtain,

$$
\left.\frac{\partial^{2} V}{\partial \epsilon \partial r}\right|_{\epsilon=0}=\frac{1}{4 \pi}\left(\frac{r^{3} \cos \theta-(3 / 2) r^{2}(2 r \cos \theta)}{r^{6}}+\frac{r^{3} \cos \theta-(3 / 2) r^{2}(2 r \cos \theta)}{r^{6}}\right) .
$$

After simplifying, we finally obtain

$$
\left.\frac{\partial^{2} V}{\partial \epsilon \partial r}\right|_{\epsilon=0}=-\frac{1}{\pi} \frac{\cos \theta}{r^{3}} .
$$


Adjusting the coefficient to normalize, we have the dipole Green’s function:

$$
G(x, y)=\frac{3}{2 \pi} \frac{\cos \theta}{r^{3}}
$$

\section{References}

1. M. Freidlin, Functional Integration and Partial Differential Equations (Princeton University Press, Princeton, New Jersey, 1985).

2. K. L. Chung, Z. Zhao, From Brownian Motion to Schrödinger's Equation (Springer-Verlag, Berlin, 1995).

3. M. E. Müller, Ann. Math. Stat. 27, 569 (1956).

4. J. A. Given, J. B. Hubbard, J. F. Douglas, J. Chem. Phys. 106, 3721 (1997).

5. C.-O. Hwang, S. Hong, J. Kim, J. Comp. Phys. 303, 331 (2015).

6. M. L. Mansfield, J. F. Douglas, E. J. Garboczi, Phys. Rev. E 64, 061401 (2001).

7. J. A. Given, C.-O. Hwang, M. Mascagni, New Directions in Statistical Physics: Econophysics, Bioinformatics, and Pattern Recognition (Springer-Verlag, 2004), "First- and Last-Passage Algorithms in Diffuosion Monte Carlo", pp. 47-68.

8. J. A. Given, C.-O. Hwang, M. Mascagni, Phys. Rev. E. 66, 056704 (2002).

9. M. Mascagni, C.-O. Hwang, Math. Comput. Simulat. 63, 93 (2003).

10. H.-X. Zhou, A. Szabo, J. F. Douglas, J. B. Hubbard, J. Chem. Phys. 100, 3821 (1994).

11. J. F. Douglas, H.-X. Zhou, J. B. Hubbard, Phys. Rev. E. 49, 5319 (1994).

12. C.-O. Hwang, M. Mascagni, T. Won, Math. Comput. Simulat. 80, 1089 (2010).

13. C.-O. Hwang, M. Mascagni, J. Korean Phys. Soc. 42, L1 (2003).

14. C.-O. Hwang, J. Korean Phys. Soc. 44, 469 (2004).

15. C.-O. Hwang, M. Mascagni, J. Appl. Phys. 95, 3798 (2004).

16. K. L. Chung, Green, Brown, and Probability (World Scientific, Singapore, 1995).

17. C.-O. Hwang, J. A. Given, Phys. Rev. E. 74, 027701 (2006).

18. B. A. Luty, J. A. McCammon, H.-X. Zhou, J. Chem. Phys. 97, 5682 (1992).

19. C. Yan, W. Cai, X. Zeng, SIAM J. Sci. Comput. 35-4, B868 (2) (2013)

20. J. R. Reitz, F. J. Milford, R. W. Christy, Foundations of Electromagnetism Theory (AddisonWesley Publishing Company, Inc., Massachusetts, 1979).

21. (Personal communication).

22. I. C. Kim, KSME Int. J. 17(4), 545 (2003). 